\title{
Responses of Soil Microorganisms and Enzymatic Activities to Alkaline Stress in Sugar Beet Rhizosphere
}

\author{
Lei Liu" ${ }^{1 \#}$, Yubo Wang", Zhijia Gai ${ }^{2}$, Dan Liu ${ }^{1}$, Peiran Wu${ }^{1}$, Bin Wang ${ }^{1}$, \\ Chunlei Zou ${ }^{1}$, Caifeng Li ${ }^{1 *}$, Fangfang Yang ${ }^{1}$ \\ ${ }^{1}$ College of Agronomy, Northeast Agricultural University, Harbin, Heilongjiang, China \\ ${ }_{2}^{2}$ Jiamusi Branch, Heilongjiang Academy of Agricultural Sciences, Jiamusi, Heilongjiang, China
}

Received: 20 November 2018

Accepted: 3 March 2019

\begin{abstract}
The objective of this study was to evaluate the effects of alkaline stress $(0,5,7$ and $9 \mathrm{~g} / \mathrm{kg} \mathrm{NaHCO}$ and $\mathrm{Na}_{2} \mathrm{CO}_{3}$, identified as $\mathrm{A} 0, \mathrm{~A} 5, \mathrm{~A} 7$ and $\mathrm{A} 9$ ) on sugar beet seedlings root growth, rhizosphere soil microbial population, and soil enzyme activities to better understand their functions and relationships The present results total root length (TRL), total root surface area (TRSA) and root volume (RV) with the increase of alkaline levels, whereas the above items were significantly improved in A7 at S2 and S3 stages. The root activity (RA), quantity of rhizosphere microorganisms (bacteria, fungi, actinomycete and total microbial) and soil enzyme (urease, alkaline phosphatase and catalase) activities were decreased at A0, A5, and A9 but significantly increasedat A7. Moreover, the value of all indexes except rhizosphere fungi quantity of tolerant cultivar under the same treatment was higher than sensitive cultivar. These results demonstrated that alkaline stress inhibited root growth and reduced whole plant biomass, however, the suitable concentration of alkaline could stimulate the growth of sugar beet seedlings and increase the activities of rhizosphere microorganisms and soil enzymes.
\end{abstract}

Keywords: Beta vulgaris L., alkaline soils, microbial biomass, soil enzyme activities

\section{Introduction}

Soil saline-alkalization is a major abiotic stress to agriculture worldwide, causing considerable damage to crop growth and loss of crop productivity [1]. More than 954 million hectares of land in the world is made

\#Contributed equally to this paper

*e-mail: licaifeng@neau.edu.cn up of sodic soil [2]. Saline-alkali land is the result of natural properties such as arid climate, neotectonic movement and human activities [3-4]. In northeastern China, approximately 3.78 million hectares of land are threatened by soil salinization and alkalization [5]. Sodic soils with high salt concentration and $\mathrm{pH}$ restrict plant growth and limit agricultural production in this area, in which $\mathrm{Na}_{2} \mathrm{CO}_{3}$ and $\mathrm{NaHCO}_{3}$ are the major limiting factors [6]. Enhanced salinity tolerance will enable more productive use of saline soil, and hence 
mechanisms involved in this ability are important areas of plant research [7].

The rhizosphere is characterized by high microbial abundance and activity which is involved in the nutrient acquisition and/or stress reduction strategies of plants [8-9]. Microorganisms are the principal drivers of all nutrient cycles, and especially for the decomposition of soil organic matter (SOM), thereby regenerating plant nutrients. Therefore, any effects by salt on microbial processes will have large connotations for SOM dynamics, ecosystem biogeochemical cycling, and plant nutrition [10-11]. Soil enzymes originate from a variety of organisms, especially fungi and bacteria, and represent the activity and diversity of microbial communities [12] because they are related to soil physiochemical characters, microbial community structure and vegetation [13-14]. Their activity reflects the functional diversity and activity of the microorganisms involved in decomposition processes, which are essential for soil functioning and soil ecosystem services [15-16]. Under the condition of soil pollution, the abundance and diversity of microbes decreased, but it was not clear how the rhizosphere microorganisms changed.

Sugar beet (Beta vulgaris L.) is used not only in the sugar industry but also in the production of bioethanol as a source of renewable energy [17]. It is breeding for adaptation to many abiotic stresses, including drought and salinity, and has been cultivated successfully in a wide range of climates on many different soils in temperate areas of the world [18]. Extensive studies have been conducted to elucidate the mechanisms by which plants themselves respond and adapt to salinity resulting from increases of salts in soils [19]. However, the linkage between rhizosphere processes and roots under salinity stress is not well understood. Therefore, in order to examine the effect of alkaline levels on microorganism and enzyme activity changes in the rhizosphere of sugar beets and to compare the effect of cultivars and triadic relationships under sodic stress has been defined as an important goal for this study and the study aimed at exploring the microorganism and soil enzyme changes of seedling rhizosphere and whether there were significant correlations among roots, microorganisms and soil enzyme changes under alkaline stress.

\section{Materials and Methods}

\section{Experimental Site, Soil and Plant Material}

The pot experiment was conducted at Northeast Agricultural University $\left(126^{\circ} 63^{\prime} \mathrm{E}, 45^{\circ} 44^{\prime} \mathrm{N}\right.$, Harbin,
China) in 2015. Physicochemical properties of the soil are shown in Table 1. The research site is in the northern temperate zone and continental monsoon area (rainy and hot during the summer; cold and arid during the winter) and the highest mean temperature was $23^{\circ} \mathrm{C}$ and the lowest was $12^{\circ} \mathrm{C}$ during the experiment. The average available accumulated temperature $\left(\geq 10^{\circ} \mathrm{C}\right)$ is $2709^{\circ} \mathrm{C}$

Two sugar beet cultivars (Beta vulgaris L.) with contrasting salt tolerance were selected as tolerant (KWS0143) and as sensitive (Beta464).

\section{Experimental Design}

Each pot was filled with $3 \mathrm{~kg}$ of soil and placed into a rain-proof shelter during the experiment. Nitrogen, phosphorus, and potassium were applied to each pot at planting with $113.41 \mathrm{mg} \cdot \mathrm{kg}^{-1} \mathrm{~N}, 85.06 \mathrm{mg} \cdot \mathrm{kg}^{-1}$ $\mathrm{P}_{2} \mathrm{O}_{5}$ and $56.71 \mathrm{mg} \cdot \mathrm{kg}^{-1} \mathrm{~K}_{2} \mathrm{O}$. Alkaline composites $\left(\mathrm{NaHCO}: \mathrm{Na}_{2} \mathrm{CO}_{3}\right.$, molar ratio 2:1) with different mass concentrations, including $0 \mathrm{~g} / \mathrm{kg}$ (A0), $5 \mathrm{~g} / \mathrm{kg}$ (A5), $7 \mathrm{~g} / \mathrm{kg}$ (A7) and $9 \mathrm{~g} / \mathrm{kg}$ (A9), were mixed with soil, and each treatment preserved ablank control with no plant species. All the fertilizer or alkaline salt were evenly mixed into soils for each treatment.

The sowing date was April 30. Two sugar beet cultivars were used in the pot experiment. The soil $\mathrm{pH}$ values of the $\mathrm{A} 0, \mathrm{~A} 5, \mathrm{~A} 7$ and $\mathrm{A} 9$ treatments were 7.22, 9.18, 9.40, and 9.56, respectively. The soil EC values of A0, A5, A7 and A9 treatments were $127.67 \mathrm{us} / \mathrm{cm}, 559.33,782.67$, and 981.67, respectively. 7 plants remained in each pot after emergence. 276 pots were prepared, and $800 \mathrm{ml}$ of water was watered in each pot. The pots were arranged in a randomized complete block (RCB) design.

\section{Sampling and Preparation}

Sugar beet seedlings were sampled from 08:00 to 10:00 h at 0 day (June 2, S1), 14 days (June 16, S2), and 28 days (June 30, S3) after the first pair of true leaves unfolded and morphological and physiological parameters were measured at each stage. At the same time, rhizosphere and bulk soil samples were collected and divided into two portions. The first portion wasused for determining soiling soil enzyme activities, and the second portion was stored at $4^{\circ} \mathrm{C}$ for analysis of microbial biomass. Dry matter weight of the whole seedling and root morphological parameters, root activity microbial biomass and soil enzyme activities were determined.

Table 1. Physico-chemical properties of soil used in the experiment.

\begin{tabular}{|c|c|c|c|c|c|c|}
\hline $\begin{array}{c}\text { Organic matter } \\
\left(\mathrm{g} \cdot \mathrm{kg}^{-1}\right)\end{array}$ & $\begin{array}{c}\text { Available nitrogen } \\
\left(\mathrm{mg} \cdot \mathrm{kg}^{-1}\right)\end{array}$ & $\begin{array}{c}\text { Available phosphorus } \\
\left(\mathrm{mg} \cdot \mathrm{kg}^{-1}\right)\end{array}$ & $\begin{array}{c}\text { Available potassium } \\
\left(\mathrm{mg} \cdot \mathrm{kg}^{-1}\right)\end{array}$ & $\begin{array}{c}\text { Soil moisture } \\
(\%)\end{array}$ & $\begin{array}{c}\mathrm{EH} \\
(\mu \mathrm{s} / \mathrm{cm})\end{array}$ \\
\hline 51.20 & 120.03 & 171.20 & 117.01 & 10.70 & 7.22 & 126.70 \\
\hline
\end{tabular}




\section{Determining Seedling Dry Matter Weight and Root Parameters}

Roots were washed with distilled water and blotted dry on filter paper. Subsequently, roots were arranged and floated on shallow water in a glass tray $(20 \mathrm{~cm} \times$ $30 \mathrm{~cm}$ ), scanned (on a Microtek Scan Maker i800), and analyzed with an image analyzer (Plant Root Analyzer Type LA-S; Wseen, China). Afterward, total root length $(\mathrm{TRL}, \mathrm{cm})$, total root surface area (TRSA, $\left.\mathrm{cm}^{2}\right)$ and total root volume (TRV, $\left.\mathrm{cm}^{3}\right)$ were determined.

Root activity (RA, $\mu \mathrm{g} \cdot \mathrm{g}^{-1} \cdot \mathrm{h}^{-1}$ ) was measured according to the triphenyl tetrazolium chloride (TTC) method [20]. The dehydrogenase activity is regarded as an index of root activity. Root activity $=$ amount of TTC reduction $(\mu \mathrm{g}) /$ fresh root weight $(\mathrm{g}) \times$ time $(\mathrm{h})$.

Five whole seedlings for each treatment were sampled to measure dry matter weight. The seedlings were heated for $15 \mathrm{~min}$ at $105^{\circ} \mathrm{C}$ and then dried to a constant weight at $80^{\circ} \mathrm{C}$, after which dry plant weight was determined.

Determining Dry Matter Weight of Soil Microbial Population and Enzymatic Activities

Enumeration of cultivable microbial populations was determined with traditional plate-dilution frequency technique on agar media in Petri plates [21]. Well mixed $0.1 \mathrm{~mL}$ samples of dilutions (Bacteria $10^{-6}-10^{-4}$, Actinomycete $10^{-5}-10^{-3}$ and fungi $10^{-3}-10^{-1}$; 3 repeats per concentration) with sterile deionized water were spread on the following media for cultivable microbe enumerations.

The number of bacteria was determined in the culture medium of beef-cream and peptone for 2-3 days. Actinomycete was determined in the culture medium of improved Gao 1 for 3-5 days, and fungi was determined in that of Martin's agar for 5-7 days [21].

Table 2. Effect of alkaline stress on dry matter weight and root activity in sugar beet seedlings.

\begin{tabular}{|c|c|c|c|c|c|}
\hline \multirow{2}{*}{ Stages } & \multirow{2}{*}{ Treatments } & \multicolumn{2}{|c|}{ DW (g) } & \multicolumn{2}{|c|}{$\mathrm{RA}\left(\mu \mathrm{g} \cdot \mathrm{g} \mathrm{h}^{-1}\right)$} \\
\hline & & KWS0143 & Beta464 & KWS0143 & Beta464 \\
\hline \multirow{8}{*}{$\mathrm{S} 1$} & A0 & $0.133 \mathrm{a}$ & $0.095 \mathrm{a}$ & $79.24 b$ & $70.83 b$ \\
\hline & A5 & $0.117 \mathrm{~b}$ & $0.082 b$ & $83.44 b$ & $76.44 \mathrm{~b}$ \\
\hline & A7 & $0.108 \mathrm{c}$ & $0.072 \mathrm{c}$ & $104.9 \mathrm{a}$ & $100.25 \mathrm{a}$ \\
\hline & A9 & $0.048 \mathrm{~d}$ & $0.032 \mathrm{~d}$ & $52.63 \mathrm{c}$ & $40.96 \mathrm{c}$ \\
\hline & \multicolumn{5}{|c|}{ ANOVA effect } \\
\hline & Alkaline (A) & $* * *$ & $* * *$ & $* * *$ & $* * *$ \\
\hline & Cultivar (C) & \multicolumn{2}{|c|}{$* * *$} & \multicolumn{2}{|c|}{$* * *$} \\
\hline & $\mathrm{A} \times \mathrm{C}$ & \multicolumn{2}{|c|}{$* * *$} & \multicolumn{2}{|c|}{ ns } \\
\hline \multirow{8}{*}{$\mathrm{S} 2$} & A0 & $0.408 \mathrm{c}$ & $0.360 \mathrm{c}$ & $134.79 b$ & $118.92 b$ \\
\hline & A5 & $0.463 b$ & $0.402 \mathrm{~b}$ & $141.33 b$ & $121.72 \mathrm{~b}$ \\
\hline & A7 & $0.632 \mathrm{a}$ & $0.575 \mathrm{a}$ & $158.14 \mathrm{a}$ & $145.07 \mathrm{a}$ \\
\hline & A9 & $0.187 \mathrm{~d}$ & $0.145 \mathrm{~d}$ & $82.51 \mathrm{c}$ & $70.83 c$ \\
\hline & \multicolumn{5}{|c|}{ ANOVA effect } \\
\hline & Alkaline (A) & $* * *$ & $* * *$ & $* * *$ & $* * *$ \\
\hline & Cultivar (C) & \multicolumn{2}{|c|}{$* * *$} & \multicolumn{2}{|c|}{$* * *$} \\
\hline & $\mathrm{A} \times \mathrm{C}$ & \multicolumn{2}{|c|}{$\mathrm{ns}$} & \multicolumn{2}{|c|}{$\mathrm{ns}$} \\
\hline \multirow{8}{*}{$\mathrm{S} 3$} & A0 & $1.034 \mathrm{~b}$ & $0.854 \mathrm{~b}$ & $153.00 \mathrm{c}$ & $125.92 \mathrm{c}$ \\
\hline & A5 & $1.043 b$ & $0.869 \mathrm{~b}$ & $170.28 \mathrm{~b}$ & $132.93 b$ \\
\hline & A7 & $1.147 \mathrm{a}$ & $0.991 \mathrm{a}$ & $189.42 \mathrm{a}$ & $156.74 \mathrm{a}$ \\
\hline & A9 & $0.370 \mathrm{c}$ & $0.257 \mathrm{c}$ & $86.71 \mathrm{~d}$ & $72.70 \mathrm{~d}$ \\
\hline & \multicolumn{5}{|c|}{ ANOVA effect } \\
\hline & Alkaline (A) & $* * *$ & $* * *$ & $* * *$ & $* * *$ \\
\hline & Cultivar (C) & \multicolumn{2}{|c|}{$* * *$} & \multicolumn{2}{|c|}{$* * *$} \\
\hline & $\mathrm{A} \times \mathrm{C}$ & \multicolumn{2}{|c|}{$\mathrm{ns}$} & \multicolumn{2}{|c|}{$* * *$} \\
\hline
\end{tabular}

Different lower-case letters mean significant differences at 0.05 level $(P<0.05) . * * * *$, and ns denote significance at $0.001,0.05$ and not significant $(P>0.05)$, respectively. The same below. 
All microbes were cultured at $30^{\circ} \mathrm{C}$. Soil urease, catalase, and alkaline phosphatase activities were determined by Tabatabai (1994) [22].

\section{Statistical Analysis}

Differences between treatments were calculated by one-way ANOVA and Duncan's multiple range tests at 0.05 level of probability. The least significant difference (LSD) multiple comparisons were conducted when there was significant difference $(P<0.05)$. All the data were statistically analyzed using SPSS software (Statistical Product and Service Solutions, Version 22.0, SPSS Inc. Chicago, IL, USA). Alkaline stress effects were considered fixed and cultivars were treated as random effects. Mean comparisons were made under Fisher's protected LSD Test at the 0.05 level of probability. Pearson correlation analysis wasalso performed to evaluate the degree and significance of the correlation.

\section{Results and Discussion}

\section{Changes in Plant Biomass and Root Growth in Seedlings}

As shown in Table 2, dry matter weight of seedling and root activity of sugar beet were influenced significantly by alkaline stress at each stage. There was significant difference between cultivars regarding dry matter weight and root activity at the S3 stage. For dry matter weight and root activity, significant interaction between alkaline concentration and cultivar was observed at the S1 stage. The dry matter weight for both sugar beet cultivars decreased with an increase in alkaline concentration at S1 stage and in A9 significantly decreased ateach stage $(P<0.05)$. These were consistent with most studies [23-25]. At S2 and S3 stages, however, dry matter weight first increased, and then decreased with an increase in alkaline concentration.

Table 3. Effect of alkaline stress on root morphology characteristics in sugar beet seedlings.

\begin{tabular}{|c|c|c|c|c|c|c|c|}
\hline & \multirow{2}{*}{ Treatments } & \multicolumn{2}{|c|}{ TRL (cm) } & \multicolumn{2}{|c|}{ TRSA $\left(\mathrm{cm}^{2}\right)$} & \multicolumn{2}{|c|}{$\mathrm{RV}\left(\mathrm{cm}^{3}\right)$} \\
\hline & & KWS0143 & Beta464 & KWS0143 & Beta464 & KWS0143 & Beta464 \\
\hline \multirow{8}{*}{$\mathrm{S} 1$} & $\mathrm{~A} 0$ & $14.96 \mathrm{a}$ & $13.36 \mathrm{a}$ & $3.29 \mathrm{a}$ & $2.59 \mathrm{ab}$ & $0.09 \mathrm{a}$ & $0.07 \mathrm{ab}$ \\
\hline & A5 & $14.05 \mathrm{ab}$ & $10.85 b$ & $2.80 \mathrm{~b}$ & $2.31 \mathrm{~b}$ & $0.08 \mathrm{ab}$ & $0.07 \mathrm{~b}$ \\
\hline & A7 & $12.12 \mathrm{c}$ & $8.15 \mathrm{c}$ & $2.37 \mathrm{c}$ & $1.71 \mathrm{c}$ & $0.07 \mathrm{c}$ & $0.05 \mathrm{c}$ \\
\hline & A9 & $3.53 \mathrm{~d}$ & $3.41 \mathrm{~d}$ & $1.02 \mathrm{~d}$ & $0.74 \mathrm{~d}$ & $0.03 \mathrm{~d}$ & $0.02 \mathrm{~d}$ \\
\hline & \multicolumn{7}{|c|}{ ANOVA effect } \\
\hline & Alkaline (A) & $* * *$ & $* * *$ & $* * *$ & $* * *$ & $* * *$ & $* * *$ \\
\hline & Cultivar (C) & \multicolumn{2}{|c|}{$* * *$} & \multicolumn{2}{|c|}{$* * *$} & \multicolumn{2}{|c|}{$* * *$} \\
\hline & $\mathrm{A} \times \mathrm{C}$ & \multicolumn{2}{|c|}{$* * *$} & \multicolumn{2}{|c|}{ ns } & \multicolumn{2}{|c|}{$*$} \\
\hline \multirow{8}{*}{ S2 } & A0 & $55.87 \mathrm{~b}$ & $44.20 \mathrm{~b}$ & $19.49 b$ & $17.09 \mathrm{~b}$ & $1.25 \mathrm{~b}$ & $0.87 \mathrm{~b}$ \\
\hline & A5 & $56.29 b$ & $47.46 \mathrm{~b}$ & $20.27 b$ & $18.17 \mathrm{~b}$ & $1.34 \mathrm{~b}$ & $0.97 b$ \\
\hline & A7 & $76.42 \mathrm{a}$ & $57.68 \mathrm{a}$ & $29.36 \mathrm{a}$ & $25.54 \mathrm{a}$ & $1.69 \mathrm{a}$ & $1.31 \mathrm{a}$ \\
\hline & A9 & $12.81 \mathrm{c}$ & $5.57 \mathrm{c}$ & $3.01 \mathrm{c}$ & $1.77 \mathrm{c}$ & $0.24 \mathrm{c}$ & $0.14 \mathrm{c}$ \\
\hline & \multicolumn{7}{|c|}{ ANOVA effect } \\
\hline & Alkaline (A) & $* * *$ & $* * *$ & $* * *$ & $* * *$ & $* * *$ & $* * *$ \\
\hline & Cultivar (C) & \multicolumn{2}{|c|}{$* * *$} & \multicolumn{2}{|c|}{$* * *$} & \multicolumn{2}{|c|}{$* * *$} \\
\hline & $\mathrm{A} \times \mathrm{C}$ & \multicolumn{2}{|c|}{ * } & \multicolumn{2}{|c|}{ * } & \multicolumn{2}{|c|}{$*$} \\
\hline \multirow{8}{*}{ S3 } & $\mathrm{A} 0$ & $114.20 \mathrm{~b}$ & $95.62 b$ & $60.10 \mathrm{~b}$ & $47.27 b$ & $2.60 \mathrm{~b}$ & $2.14 b$ \\
\hline & A5 & $104.81 \mathrm{c}$ & $85.48 \mathrm{c}$ & $55.05 \mathrm{~b}$ & $45.76 b$ & $2.49 \mathrm{c}$ & $2.01 \mathrm{c}$ \\
\hline & A7 & $124.88 \mathrm{a}$ & $104.73 a$ & $67.34 \mathrm{a}$ & $54.76 \mathrm{a}$ & $2.90 \mathrm{a}$ & $2.46 \mathrm{a}$ \\
\hline & A9 & $23.89 \mathrm{~d}$ & $8.55 \mathrm{~d}$ & $5.70 \mathrm{c}$ & $4.06 \mathrm{c}$ & $0.47 \mathrm{~d}$ & $0.27 \mathrm{~d}$ \\
\hline & \multicolumn{7}{|c|}{ ANOVA effect } \\
\hline & Alkaline (A) & $* * *$ & $* * *$ & $* * *$ & $* * *$ & $* * *$ & $* * *$ \\
\hline & Cultivar (C) & \multicolumn{2}{|c|}{$* * *$} & \multicolumn{2}{|c|}{$* * *$} & \multicolumn{2}{|c|}{$* * *$} \\
\hline & $\mathrm{A} \times \mathrm{C}$ & \multicolumn{2}{|c|}{ ns } & \multicolumn{2}{|c|}{ * } & \multicolumn{2}{|c|}{$* *$} \\
\hline
\end{tabular}




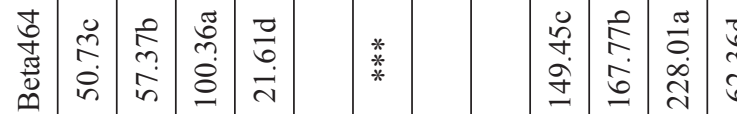

पू $\widehat{60}$

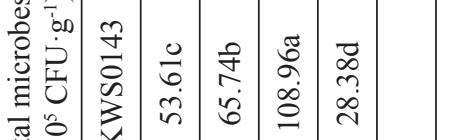
퐁

泀

音

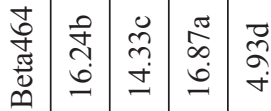

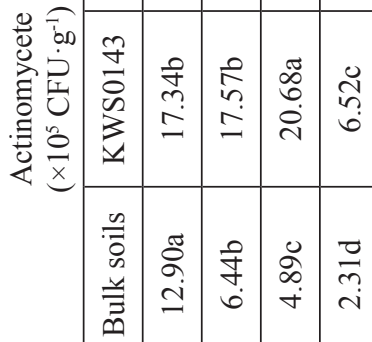

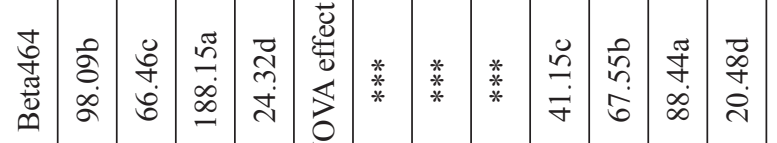

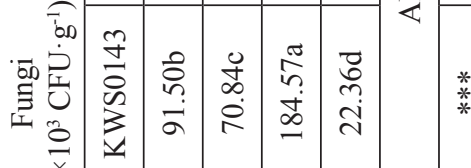

:

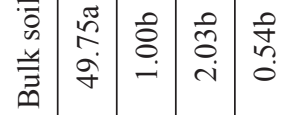

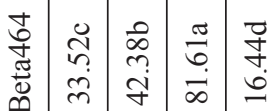

क.

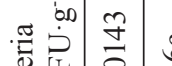

竎

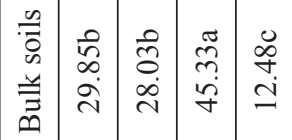

䔍

\begin{tabular}{|c|c|c|c|}
\hline * & $\begin{array}{l}\mathscr{E} \\
\stackrel{0}{\Sigma} \\
\underline{I}\end{array}$ & $\begin{array}{l}\stackrel{े}{m} \\
\stackrel{े}{े}\end{array}$ & 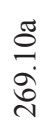 \\
\hline & 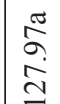 & $\begin{array}{l}\ddot{0} \\
\stackrel{0}{\approx} \\
\stackrel{1}{*}\end{array}$ & 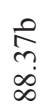 \\
\hline
\end{tabular}

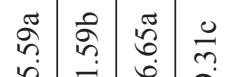

ये $\bar{\sim}$ ¿ें

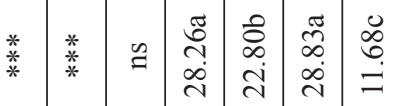

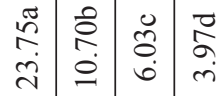

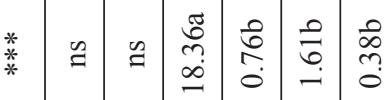

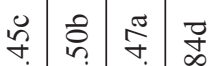

ปี่

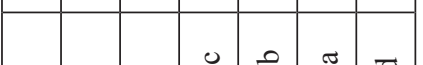

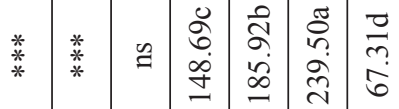

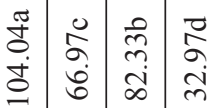

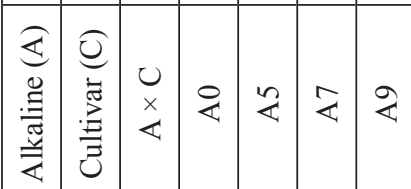

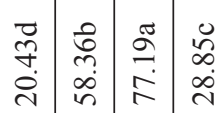

\begin{tabular}{|c|c|c|c|}
\hline & $\begin{array}{l}0 \\
\tilde{b} \\
i \\
\Xi\end{array}$ & 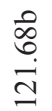 & 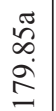 \\
\hline & $\begin{array}{l}0 \\
\bar{n} \\
\grave{I}\end{array}$ & 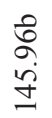 & 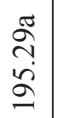 \\
\hline & $\begin{array}{l}\tilde{D} \\
\infty \\
\infty \\
\infty \\
\infty\end{array}$ & $\frac{0}{\hat{c}}$ & 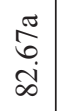 \\
\hline & $\mid \begin{array}{c}\frac{\pi}{0} \\
\stackrel{9}{m} \\
m\end{array}$ & $\begin{array}{l}0 \\
0 \\
\\
\end{array}$ & 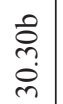 \\
\hline
\end{tabular}

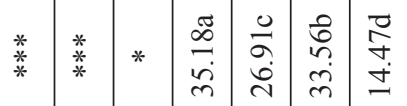

* * $\stackrel{\text { * }}{*}$

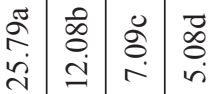

\begin{tabular}{|c|c|c|c|c|c|c|c|c|c|c|}
\hline 苂 & \begin{tabular}{|l}
$*$ \\
$*$ \\
$*$
\end{tabular} & $g$ & 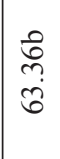 & $\begin{array}{l}\vec{n} \\
\infty \\
\stackrel{+}{r}\end{array}$ & $\left|\begin{array}{l}\mid \pi \\
2 \\
\infty \\
\infty \\
\infty\end{array}\right|$ & $\frac{\dot{b}}{\dot{0}}$ & 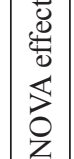 & * & $\underset{*}{*}$ & $\mathscr{q}$ \\
\hline
\end{tabular}

*

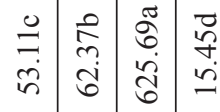

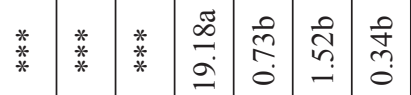

* $\stackrel{*}{*} \underset{*}{*} * \frac{*}{*}$

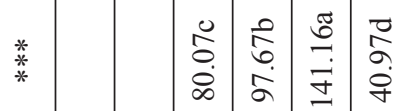

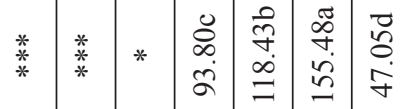

* $*$ *

*

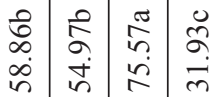

ङ 0

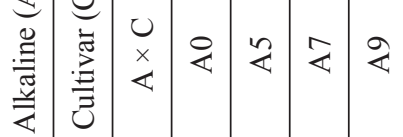

ङ 0

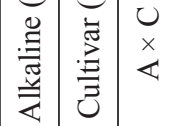


Table 5. Effect of alkaline stress on soil enzyme activities in sugar beet rhizosphere and bulk soil.

\begin{tabular}{|c|c|c|c|c|c|c|c|c|c|c|}
\hline \multirow[t]{2}{*}{ Stages } & \multirow{2}{*}{ Treatments } & \multicolumn{3}{|c|}{ Urease $\left(\mathrm{mg} \mathrm{NH}_{4}^{+}-\mathrm{N} \cdot \mathrm{g}^{-1} \cdot \mathrm{h}^{-1}\right)$} & \multirow[b]{2}{*}{ Bulk soils } & \multirow[b]{2}{*}{ KWS0143 } & \multirow[b]{2}{*}{ Beta464 } & \multicolumn{3}{|c|}{$\begin{array}{c}\text { Catalase }\left(\mathrm{mL} 0.1 \mathrm{~mol} \cdot \mathrm{L}^{-1}\right. \\
\left.\mathrm{KMnO}_{4} \cdot \mathrm{g}^{-1}\right)\end{array}$} \\
\hline & & Bulk soils & KWS0143 & Beta464 & & & & Bulk soils & KWS0143 & Beta464 \\
\hline \multirow{8}{*}{ S1 } & $\mathrm{A} 0$ & $0.67 \mathrm{~b}$ & $0.88 \mathrm{~b}$ & $0.80 \mathrm{~b}$ & $0.28 \mathrm{c}$ & $0.43 \mathrm{a}$ & $0.48 \mathrm{a}$ & $2.28 \mathrm{a}$ & $2.72 \mathrm{a}$ & $2.60 \mathrm{a}$ \\
\hline & A5 & $0.67 \mathrm{~b}$ & $0.93 b$ & $0.89 \mathrm{~b}$ & $0.31 \mathrm{~b}$ & $0.35 \mathrm{c}$ & $0.35 \mathrm{c}$ & $1.28 \mathrm{~b}$ & $2.20 \mathrm{~b}$ & $2.04 \mathrm{c}$ \\
\hline & A7 & $0.76 \mathrm{a}$ & $1.44 \mathrm{a}$ & $1.24 \mathrm{a}$ & $0.35 \mathrm{a}$ & $0.39 b$ & $0.39 \mathrm{~b}$ & $1.08 \mathrm{bc}$ & $2.76 \mathrm{a}$ & $2.36 \mathrm{ab}$ \\
\hline & A9 & $0.46 \mathrm{c}$ & $0.50 \mathrm{c}$ & $0.50 \mathrm{c}$ & $0.18 \mathrm{~d}$ & $0.22 \mathrm{~d}$ & $0.20 \mathrm{~d}$ & $0.92 \mathrm{c}$ & $1.12 \mathrm{c}$ & $0.96 \mathrm{~d}$ \\
\hline & \multicolumn{10}{|c|}{ ANOVA effect } \\
\hline & Alkaline (A) & $* *$ & $* * *$ & $* * *$ & $* * *$ & $* * *$ & $* * *$ & $* * *$ & $* * *$ & $* * *$ \\
\hline & Cultivar (C) & & $* *$ & & $\mathrm{~ns}$ & & $* *$ & & & \\
\hline & $\mathrm{A} \times \mathrm{C}$ & & $\mathrm{ns}$ & & $* *$ & & $\mathrm{~ns}$ & & & \\
\hline \multirow{8}{*}{ S2 } & A0 & $0.97 \mathrm{a}$ & $1.42 \mathrm{~b}$ & $1.28 \mathrm{~b}$ & $0.22 \mathrm{c}$ & $0.40 \mathrm{~b}$ & $0.39 b$ & $1.84 \mathrm{a}$ & $2.08 \mathrm{a}$ & $2.16 \mathrm{a}$ \\
\hline & A5 & $0.83 b$ & $1.39 \mathrm{~b}$ & $1.29 \mathrm{~b}$ & $0.26 b$ & $0.43 b$ & $0.34 \mathrm{c}$ & $0.92 b$ & $1.68 \mathrm{c}$ & $1.56 \mathrm{c}$ \\
\hline & A7 & $0.99 \mathrm{a}$ & $1.96 \mathrm{a}$ & $1.80 \mathrm{a}$ & $0.29 a$ & $0.51 \mathrm{a}$ & $0.47 \mathrm{a}$ & $0.72 \mathrm{c}$ & $1.92 \mathrm{~b}$ & $1.68 \mathrm{~b}$ \\
\hline & A9 & $0.53 \mathrm{c}$ & $0.74 \mathrm{c}$ & $0.58 \mathrm{c}$ & $0.14 \mathrm{~d}$ & $0.29 \mathrm{c}$ & $0.23 \mathrm{~d}$ & $0.52 \mathrm{~d}$ & $1.00 \mathrm{~d}$ & $0.64 \mathrm{~d}$ \\
\hline & \multicolumn{10}{|c|}{ ANOVA effect } \\
\hline & Alkaline (A) & $* *$ & $* * *$ & $* * *$ & $* *$ & $* * *$ & $* * *$ & $* * *$ & $* * *$ & $* * *$ \\
\hline & Cultivar (C) & & $* * *$ & & $* * *$ & & $* * *$ & & & \\
\hline & $\mathrm{A} \times \mathrm{C}$ & & $\mathrm{ns}$ & & * & & $* *$ & & & \\
\hline \multirow{8}{*}{ S3 } & A0 & $0.86 \mathrm{ab}$ & $1.15 \mathrm{c}$ & $1.06 \mathrm{~b}$ & $0.24 b$ & $0.36 \mathrm{c}$ & $0.33 b$ & $2.24 \mathrm{a}$ & $2.72 \mathrm{a}$ & $2.48 \mathrm{a}$ \\
\hline & A5 & $0.75 b$ & $1.28 \mathrm{~b}$ & $1.06 \mathrm{~b}$ & $0.26 \mathrm{~b}$ & $0.41 \mathrm{~b}$ & $0.34 \mathrm{~b}$ & $1.28 \mathrm{~b}$ & $2.36 \mathrm{c}$ & $2.08 \mathrm{c}$ \\
\hline & A7 & $0.93 \mathrm{a}$ & $1.82 \mathrm{a}$ & $1.60 \mathrm{a}$ & $0.29 \mathrm{a}$ & $0.58 \mathrm{a}$ & $0.55 \mathrm{a}$ & $1.08 \mathrm{c}$ & $2.60 \mathrm{~b}$ & $2.36 \mathrm{~b}$ \\
\hline & A9 & $0.42 \mathrm{c}$ & $0.58 \mathrm{~d}$ & $0.49 \mathrm{c}$ & $0.13 \mathrm{c}$ & $0.20 \mathrm{~d}$ & $0.16 \mathrm{c}$ & $0.92 \mathrm{c}$ & $1.56 \mathrm{~d}$ & $1.20 \mathrm{~d}$ \\
\hline & \multicolumn{10}{|c|}{ ANOVA effect } \\
\hline & Alkaline (A) & $* *$ & $* * *$ & $* * *$ & $* * *$ & $* * *$ & $* * *$ & $* * *$ & $* * *$ & $* * *$ \\
\hline & Cultivar (C) & & $* * *$ & & $* * *$ & & $* * *$ & & & \\
\hline & $\mathrm{A} \times \mathrm{C}$ & & ns & & ns & & ns & & & \\
\hline
\end{tabular}

The A7 treatment significantly increased dry matter weight per plant of two cultivars $(P<0.05)$. The same conclusion has been reported in Chenopodiaceae, such as Kochia sieversiana [26], Salsolanitraria Pall, Haloxylonammodendron [27], etc. Zou et al. (2017) reported that low levels of alkaline stress significantly promoted the growth of sugar beet due to the strong salt tolerance [28]. Interestingly, the root activities of both sugar beet cultivars first increased, and then declined withan increase inalkaline concentration at each stage in our study. This probably could be caused by the activation of $\mathrm{Na}^{+}$transporters by salinity and the enhancement of root viability to adapt to the stress environment [29].

The total root length, surface area and root volume decreased in alkaline conditions at S1 stage (Table 3), which indicated that the growth of new roots was hampered. A similar result was also found in Arachishypogaea plants [30]. However, it increased obviously under A7 alkaline stress compared to A0 at the S3 stage, which suggested that beet roots adapted to A7 alkaline stress [31]. Moreover, the total root length, surface area,root volume,dry matter weight and root activities values of KWS 0143 in A5, A7 and A9 were higher than those of Beta 464 at each stage.

\section{Changes in Rhizosphere Microorganisms of Seedlings}

As shown in Table 4, the number of microbes in the rhizosphere soil were significantly influenced by alkaline stress at each stage. There was significant difference between cultivars for microbial populations at stages S2 and S3. However, there was no significant interaction between alkaline concentration and cultivar 
Table 6. Correlation coefficients between root parameters and other environmental factors under alkaline stress of KWS0143.

\begin{tabular}{|c|c|c|c|c|c|c|}
\hline Stages & Parameter & Root activity & $\begin{array}{l}\text { Bacteria } \\
\text { quantity }\end{array}$ & Fungi quantity & $\begin{array}{l}\text { Actinomycete } \\
\text { quantity }\end{array}$ & $\begin{array}{l}\text { Total microbial } \\
\text { quantity }\end{array}$ \\
\hline \multirow{9}{*}{ S1 } & Dry weight & 0.695 & 0.407 & 0.526 & 0.877 & 0.509 \\
\hline & Root activity & 1 & 0.94 & 0.946 & $0.954^{*}$ & $0.973^{*}$ \\
\hline & Total root length & 0.713 & 0.431 & 0.523 & 0.89 & 0.531 \\
\hline & Surface area & 0.601 & 0.291 & 0.425 & 0.811 & 0.399 \\
\hline & Root volume & 0.642 & 0.342 & 0.444 & 0.842 & 0.447 \\
\hline & Urease activity & $0.985^{*}$ & $0.978^{*}$ & $0.981 *$ & 0.892 & $0.994 * *$ \\
\hline & Phosphatase activity & 0.766 & 0.519 & 0.685 & 0.899 & 0.609 \\
\hline & Catalase activity & 0.877 & 0.68 & 0.814 & $0.955^{*}$ & 0.755 \\
\hline & Total microbial quantity & $0.973^{*}$ & $0.993 * *$ & $0.963^{*}$ & 0.859 & 1 \\
\hline \multirow{9}{*}{$\mathrm{S} 2$} & Dry weight & $0.972 *$ & $0.996^{* *}$ & 0.789 & 0.868 & $0.997 * *$ \\
\hline & Root activity & 1 & $0.971 *$ & 0.665 & 0.93 & $0.981 *$ \\
\hline & Total root length & $0.995^{* *}$ & $0.970^{*}$ & 0.657 & 0.946 & $0.981 *$ \\
\hline & Surface area & $0.991 * *$ & $0.980^{*}$ & 0.695 & 0.929 & $0.989 *$ \\
\hline & Root volume & $1.000^{* *}$ & $0.969^{*}$ & 0.657 & 0.937 & $0.980^{*}$ \\
\hline & Urease activity & $0.962 *$ & $0.971 *$ & 0.715 & 0.909 & $0.979 *$ \\
\hline & Phosphatase activity & $0.974 *$ & $0.997 * *$ & 0.791 & 0.867 & $0.999 * *$ \\
\hline & Catalase activity & 0.878 & 0.751 & 0.238 & $0.986^{*}$ & 0.784 \\
\hline & Total microbial quantity & $0.981 *$ & $0.999 * *$ & 0.789 & 0.866 & 1 \\
\hline \multirow{9}{*}{$\mathrm{S} 3$} & Dry weight & $0.978 *$ & 0.883 & 0.532 & 0.929 & 0.876 \\
\hline & Root activity & 1 & $0.957^{*}$ & 0.646 & 0.852 & $0.950^{*}$ \\
\hline & Total root length & $0.961 *$ & 0.860 & 0.534 & $0.960^{*}$ & 0.855 \\
\hline & Surface area & $0.964 *$ & 0.865 & 0.540 & $0.957^{*}$ & 0.86 \\
\hline & Root volume & $0.969^{*}$ & 0.869 & 0.53 & 0.947 & 0.863 \\
\hline & Urease activity & 0.913 & $0.981 *$ & 0.901 & 0.745 & $0.987 *$ \\
\hline & Phosphatase activity & 0.946 & $0.994 * *$ & 0.858 & 0.766 & $0.996^{* *}$ \\
\hline & Catalase activity & 0.875 & 0.736 & 0.426 & $0.994 * *$ & 0.734 \\
\hline & Total microbial quantity & $0.950 *$ & $0.999 * *$ & 0.839 & 0.732 & 1 \\
\hline
\end{tabular}

Data in the table are r-values. * and ** represent correlation at 0.05 and 0.01 , respectively.

for bacteria and total microbial quantity at $\mathrm{S} 1$ or $\mathrm{S} 3$. The rhizosphere soil showed significantly higher the number of the bacteria and fungi than the bulk soil, particularly at stages S2 and S3. The bacteria and total microbial quantity in the rhizosphere soil of both cultivars first increased, and then decreased with increasing alkaline concentration. Several studies have reported $\mathrm{pH}$ to be a major factor influencing community structures across soil habitats [32]. According to our results, the quantities of both cultivars rhizosphere bacteria in A7 were 61.07-144.44\% higher than A0 treatment at each stage. It may be that plants release root exudate to the rhizosphere in order to absorb the large amounts of PGPR to feed themselves under alkaline stress [33]. Moreover, research suggests that Arbuscular Mycorrhizae (AM) could increase total root surface area and make plants absorb water and nutrients more effectively, thus improving plant stress resistance [34]. The quantity of both cultivars rhizosphere fungi in A7 were $91.80 \sim 1224.09 \%$ higher than A0 at all stages in this study. This may be caused by the combination of beet roots and symbiotic fungi. The bacteria, actinomycete and total microbial quantity for KWS0143 with A9 were higher than Beta464 at each stage, but that was the opposite for fungi quantity. 
Table 7. Correlation coefficients between root parameters and other environmental factors under alkaline stress of Beta464.

\begin{tabular}{|c|c|c|c|c|c|c|}
\hline & Parameter & Root activity & Bacteria quantity & Fungi quantity & $\begin{array}{l}\text { Actinomycete } \\
\text { quantity }\end{array}$ & $\begin{array}{c}\text { Total microbial } \\
\text { quantity }\end{array}$ \\
\hline \multirow{9}{*}{ S1 } & Dry weight & 0.633 & 0.373 & 0.456 & 0.908 & 0.481 \\
\hline & Root activity & 1 & $0.953^{*}$ & 0.924 & 0.892 & $0.981^{*}$ \\
\hline & Total root length & 0.490 & 0.208 & 0.311 & 0.824 & 0.324 \\
\hline & Surface area & 0.529 & 0.248 & 0.320 & 0.839 & 0.360 \\
\hline & Root volume & 0.548 & 0.269 & 0.325 & 0.844 & 0.379 \\
\hline & Urease activity & $0.992 * *$ & $0.983 *$ & 0.942 & 0.831 & $0.997 * *$ \\
\hline & Phosphatase activity & 0.660 & 0.443 & 0.606 & 0.918 & 0.546 \\
\hline & Catalase activity & 0.792 & 0.598 & 0.714 & $0.978 *$ & 0.690 \\
\hline & Total microbial quantity & $0.981 *$ & $0.993 * *$ & $0.961 *$ & 0.801 & 1 \\
\hline \multirow{9}{*}{ S2 } & Dry weight & $0.985^{*}$ & $0.999 * *$ & $0.961 *$ & 0.892 & $0.999 * *$ \\
\hline & Root activity & 1 & $0.982 *$ & 0.919 & $0.951^{*}$ & $0.991 * *$ \\
\hline & Total root length & $0.990 * *$ & $0.954 *$ & 0.889 & $0.963^{*}$ & $0.967 *$ \\
\hline & Surface area & $1.000 * *$ & $0.982 *$ & 0.922 & 0.950 & $0.991 * *$ \\
\hline & Root volume & $0.999 * *$ & $0.986^{*}$ & 0.934 & 0.939 & $0.993 * *$ \\
\hline & Urease activity & $0.992 * *$ & $0.990 *$ & 0.929 & 0.927 & $0.995^{* *}$ \\
\hline & Phosphatase activity & $0.961 *$ & 0.938 & 0.835 & 0.945 & $0.951^{*}$ \\
\hline & Catalase activity & 0.782 & 0.649 & 0.482 & 0.933 & 0.69 \\
\hline & Total microbial quantity & $0.991 * *$ & $0.998 * *$ & $0.960 *$ & 0.903 & 1 \\
\hline \multirow{9}{*}{ S3 } & Dry weight & $0.982 *$ & 0.887 & 0.541 & 0.912 & 0.874 \\
\hline & Root activity & 1 & $0.959^{*}$ & 0.685 & 0.865 & $0.951^{*}$ \\
\hline & Surface area & $0.974 *$ & 0.870 & 0.525 & 0.932 & 0.857 \\
\hline & Root volume & $0.974 *$ & 0.872 & 0.537 & 0.941 & 0.86 \\
\hline & Total microbial quantity & $0.951 *$ & $0.999 * *$ & 0.867 & 0.727 & 1 \\
\hline & Urease activity & 0.945 & $0.984 *$ & 0.882 & 0.787 & $0.989^{*}$ \\
\hline & Phosphatase activity & 0.949 & $0.988^{*}$ & 0.878 & 0.782 & $0.992 * *$ \\
\hline & Catalase activity & 0.889 & 0.742 & 0.416 & $0.991 * *$ & 0.732 \\
\hline & Total root length & $0.961 *$ & 0.847 & 0.508 & $0.954 *$ & 0.835 \\
\hline
\end{tabular}

\section{Changes of Rhizosphere Soil Enzyme Activities of Seedlings}

Soil enzymes are the mediator and catalysts of most soil transformation processes. As shown in Table 5, soil enzyme activities varied significantly in response to alkaline stress at each stage. Significant interactions between alkaline concentration and cultivar are observed for urease at each stage, alkaline phosphatase at S3 stage and catalase activity at stages S1 or S3. Results from our experiment indicated that high $\mathrm{pH}$ decreased activities of soil enzymes. Hendriksen (2016) [16] also found that the activity of the enzymes depend on $\mathrm{pH}$. The A7 treatment produced the highest values for soil enzyme activities of both cultivars at each stage, while A9 treatment gave the lowest value for the soil enzymes. The urease, alkaline phosphatase and catalase activities showed that similar changes among treatments and activitiesof KWS0143 were higher at later stages (S2 and S3).

\section{Relationships between Root Parameters and Other Indexes}

The correlation among traits of two varieties was shown in Tables 6 and 7, respectively. Significantly positive correlation among bacteria quantity, total microbial quantity and urease activity was observed at all stages. Similarly, Eriksson found that enzyme is mainly derived from microbial population, in particular 
bacteria, and they concluded that the phenomenon might have been due to the key role of both bacteria and urease in nutrient cycling [35]. Soil enzymes are synthetized and secreted by soil microorganisms, and are the proximate agents of organic matter formation and decomposition [36].

Soil enzymes may also be affected indirectly by plant microbe interactions. There was positively significant relationship between root activity and dry weight at stages S2 and S3. Moreover, there was positive relationship between root activity and total microbial quantity at S1, S2, and S3. With the increase of alkaline concentration, dry matter of seedling and root growth decreased at the S1 stage, whereas it increased and then decreased at the later seedling stage, while root activity kept increasing and then decreasing at the whole seedling stage. These results indicate that succinate dehydrogenase, which represents the activity of beet root, is an important factor in the formation of rhizosphere microbial communities [37]. The beet roots could release exudates to attract probiotics in the adaptive phase under alkaline stress [38].

Under suitable alkaline concentration, the number of rhizospheric microorganisms is increased. Among them, fungi increased the order of magnitude, but the number of bacteria was still the largest, dominating the whole microbial community. From the point of view of time, the effect of anaphase is more relevant, which may be related to root growth and elongation [29]. The cause of the correlation between the root activity of the beet and the enzyme is probably the role of the bacteria, but the function of fungi could not be ignored. Studies have also shown that fungi play an important role in alkaline conditions [39-41]. Urease activity comes from microbial population and the increased urease also provides nutrients for plants. Further research is required to study in detail the interactions between the root environment and microorganisms, and how such interactions affect the activities of soil enzymes [42].

\section{Conclusions}

In the present study, we evaluated the effects of different levels of alkaline on growth of sugar beet and soil enzyme activity in the rhizosphere soil. Our results indicate that a high level (A9) of alkaline stress significantly decreased dry matter accumulation, root morphology, root activity, soil microbe quantity and enzyme activity at late seedling stage, whereas middle level (A7) of alkaline stress increased all of these parameters at late seedling stage, especially root activity, rhizosphere bacteria, fungi and urease activity. Dry matter weightand root activity of KWS0143 under alkaline stress were higher compared with Beta464. The results can provide theoretical reference for improving sugar beet saline alkali soil.

\section{Acknowledgements}

Special thanks to the National Natural Science Foundation of China (grant No. 31671622). We also thank Ying Liu for her encouragement.

\section{Conflict of Interest}

The authors declare no conflict of interest.

\section{References}

1. MART N.M.R, IGLESIAS D.J., FORNER-GINER M.A., PRIMO-MILLO E., LEGAZ F. The effect of sodium bicarbonate on plant performance and iron acquisition system of FA-5 (Forner-Alcaide 5) citrus seedlings. Acta Physiologiae Plantarum. 35 (9), 2833, 2013.

2. ZHANG X., TAKANO T., LIU S. Identification of a mitochondrial ATP synthase small subunit gene (RMtATP6) expressed in response to salts and osmotic stresses in rice (Oryza sativa L.). Journal of Experimental Botany. 57, 193, 2006.

3. BIAN J., TANG J., LIN N.F. Relationship between saline-alkali soil formation and neotectonic movement in Songnen Plain, China. Environmental Geology. 55 (7), $1421,2008$.

4. YANG J., ZHANG S.W., YING L.I., KUN B. U., ZHANG Y.B., CHANG L.P., ZHANG Y.Z. Dynamics of salinealkali land and its ecological regionalization in western songnen plain, China. Chinese Geographical Science. 20 (2), 159, 2010.

5. YANG C.W., SHI D.C., WANG D.L.. Comparative effects of salt and alkali stresses on growth, osmotic adjustment and ionic balance of an alkali-resistant halophyte Suaeda glauca (Bge.). Plant Growth Regulation. 56 (2), 179, 2008.

6. YANGZ., WANG B.S. Present status of saline soil resources and countermeasures for improvement and utilization in China. Shandong Agricultural Sciences. 2015 (4), 125, 2015.

7. KATORI T., IKEDA A., IUCHI S., KOBAYASHI M., SHINOZAKI K., MAEHASHI K., SAKATA Y., TANAKA S., TAJI T. Dissecting the genetic control of natural variation in salt tolerance of Arabidopsis thaliana accessions. Journal of Experimental Botany. 61 (4), 1125, 2010.

8. KAUR C., SELVAKUMAR G., GANESHAMURTHY A.N. Organic Acids in the Rhizosphere: Their Role in Phosphate Dissolution. Springer India: Microbial Inoculants in Sustainable Agricultural Productivity. 2016.

9. JONES D. Organic acids in the rhizosphere - a critical review. Plant and Soil. 205 (1), 25, 1998.

10. SETIA R., MARSCHNER P. Erratum to: Carbon mineralization in saline soils as affected by residue composition and water potential. Biology \& Fertility of Soils. 49 (6), 777, 2013.

11. RAICH J.W., POTTER C.S. Global patterns of carbon dioxide emissions from soils. Global Biogeochemical Cycles. 9 (1), 23, 1995.

12. BASTIDA F., ZSOLNAY A., HERN N.T., GARC A.C. Past, present and future of soil quality indices: A biological perspective. Geoderma. 147 (3), 159, 2008. 
13. KITAYAMA K. The activities of soil and root acid phosphatase in the nine tropical rain forests that differ in phosphorus availability on Mount Kinabalu, Borneo. Plant and Soil. 367 (1), 215, 2013.

14. CORBIN J.D., D'ANTONIO C.M. Gone but Not Forgotten? Invasive Plants' Legacies on Community and Ecosystem Properties. Invasive Plant Science \& Management. 5 (1), 117, 2016.

15. SINSABAUGH R., LAUBER Cl., WEINTRAUB M., AHMED B., ALLISON S., CRENSHAW C., CONTOSTA A., CUSACK D., FREY S., GALLO M. Stoichiometry of soil enzyme activity at global scale. Ecology Letters. 11 (11), 1252, 2010.

16. HENDRIKSEN N.B., CREAMER R.E., STONE D., WINDING A. Soil exo-enzyme activities across Europe - The influence of climate, land-use and soil properties. Applied Soil Ecology. 97, 44, 2016.

17. MAGA A.C., N EZ-S N.N., FERN N.S.V.M., GARC A.P., SERRANO A., P REZ-MAR N.D., PEM N J.M., ALCALDE E. Direct prediction of bioethanol yield in sugar beet pulp using near infrared spectroscopy. Bioresource Technology. 102 (20), 9542, 2011.

18. VASTARELLI P., MOSCHELLA A., PACIFICO D., MANDOLINO G. Water stress in Beta vugaris: osmotic adjustment response and gene expression analysis in ssp. vulgaris and marittima. American Journal of Plant Sciences. 4 (1), 11, 2013.

19. WANG H., WU Z., CHEN Y., YANG C., SHI D. Effects of salt and alkali stresses on growth and ion balance in rice (Oryza sativa L.). Plant, Soil and Environment - UZEI (Czech Republic). 57 (6), 286, 2011.

20. BERRY J.A., BEERLING D.J., FRANKS P.J. Stomata: key players in the earth system, past and present. Current Opinion in Plant Biology. 13 (3), 232, 2010.

21. HARRIS R.F., SOMMERS L.E. Plate-dilution frequency technique for assay of microbial ecology. Applied Microbiology. 16 (2), 330, 1968.

22. WEAVER R.W., MICKELSON S.H. Microbiological and biochemical properties. Soil Science Society of America. 1994.

23. BOR M., ÖZDEMIR F., TÜRKAN I. The effect of salt stress in lipid peroxidation and antioxidants in leaves of sugar beet Beta vulgaris L. and wild beet Beta maritima $\mathrm{L}$. Plant Science 164 (1), 77, 2003.

24. WAKEEL A., ASIF A.R., PITANN B., SCHUBERT S. Proteome analysis of sugar beet (Beta vulgaris L.) elucidates constitutive adaptation during the first phase of salt stress. Journal of Plant Physiology. 168 (6), 519, 2011.

25. WANG Y.G., STEVANATO P., YU L.H., ZHAO H.J, SUN X.W., SUN F., LI J., GENG G. The physiological and metabolic changes in sugar beet seedlings under different levels of salt stress. Journal of Plant Research. 130 (6), 1079, 2017.

26. YAN Y.Q., LIU X.L., WANGK., FAN J.P., SHI X.C. Effect of complex saline-alkali stress on physiological parameters of Nitratia tangutorum. Chinese Journal of Plant Ecology. 34 (10), 1213, 2010.

27. YI L.G., MA J., LI Y. Characteristics and vitality of the roots in the seedling stage in the three desert plants under salt stress. Science in China (Earth Sciences). 36 (2), 86, 2006.
28. ZOU C.L., SANG L.M., GAI Z.J., WANG Y.B., LI C.F. Morphological and Physiological Responses of Sugar Beet to Alkaline Stress. Sugar Tech. 20 (2), 202, 2018.

29. VERA-ESTRELLA R., BARKLA B.J., GARC A-RAM R.L., PANTOJA O. Salt stress in Thellungiella halophila activates $\mathrm{Na}+$ transport mechanisms required for salinity tolerance. Plant Physiology. 139 (3), 1507, 2005.

30. YU T.Y., WANG C.X., SUN X.W., SUN X.S., ZHENG Y.M., WU Z.F., SHEN P., WANG C.B. Effects of alkaline stress on root morphology and dry matter accumulation characteristics of peanut seedling. Chinese Journal of Oil Crop Sciences. 2 (39), 190, 2017.

31. CHENGJIANG R., SILVA J.A.T.D, MOPPER S., PEI Q., LUTTS S. Halophyte improvement for a salinized world. Critical Reviews in Plant Sciences. 29 (6), 329, 2010.

32. TRIPATHI B.M., KIM M., TATENO R., KIM W., WANG J.J., LAI-HOE A., SHUKOR N.A.A., RAHIM R.A., GO R., ADAMS J.M. Soil $\mathrm{pH}$ and biome are both key determinants of soil archaeal community structure. Soil Biology \& Biochemistry. 88, 1, 2015.

33. CHEN X., TANG J.J., FANG Z.G., SHIMIZU K. Effects of weed communities with various species numbers on soil features in subtropical orchard ecosystem. Agriculture Ecosystems \& Environment. 102 (3), 377, 2004.

34. EVELIN H., KAPOOR R., GIRI B. Arbuscular mycorrhizal fungi in alleviation of salt stress: a review. Annals of Botany. 104 (7), 1263, 2009.

35. ERIKSSON M., KA J.O, MOHN W.W. Effects of Low Temperature and Freeze-Thaw Cycles on Hydrocarbon Biodegradation in Arctic Tundra Soil. Applied \& Environmental Microbiology. 67 (11), 5107, 2001.

36. BURNS R.G., DEFOREST J.L., MARXSEN J., SINSABAUGH R.L., STROMBERGER M.E., WALLENSTEIN M.D., WEINTRAUB M.N., ZOPPINI A. Soil enzymes in a changing environment: Current knowledge and future directions. Soil Biology \& Biochemistry. 58 (2), 216, 2013.

37. BERG G., SMALLA K. Plant species and soil type cooperatively shape the structure and function of microbial communities in the rhizosphere. FEMS Microbiology Ecology. 68 (1), 1, 2009.

38. BUWALDA J. G. The carbon costs of root systems of perennial fruit crops. Environmental \& Experimental Botany. 33 (1), 131, 1993.

39. KHALIL H.A., EISSA A.M., EL-SHAZLY S.M., NASR A.M. Aboul. Improved growth of salinity-stressed citrus after inoculation with mycorrhizal fungi. Scientia Horticulturae. 130 (3), 624, 2011.

40. HARLEY J.L., SMITH S.E. Mycorrhizal symbiosis. Quarterly Review of Biology. 3 (3), 273, 2008.

41. SMITH S.E., JAKOBSEN I., GRONLUND M., SMITH F.A. Roles of arbuscular mycorrhizas in plant phosphorus nutrition: interactions between pathways of phosphorus uptake in arbuscular mycorrhizal roots have important implications for understanding and manipulating plant phosphorus acquisition. Plant Physiology. 156 (3), 1050, 2011.

42. WANG Y.F., FU F.Y., LI J.J., WANG G.S., WU M.M., ZHAN J., CHEN X.S., MAO Z.Q. Effects of seaweed fertilizer on the growth of Malus hupehensis Rehd. seedlings, soil enzyme activities and fungal communities under replant condition. European Journal of Soil Biology. 75, 1, 2016. 